\title{
Safety and Tumor Responses with Lambrolizumab (Anti-PD-1) in Melanoma
}

\author{
Omid Hamid, M.D., Caroline Robert, M.D., Ph.D., Adil Daud, M.D., \\ F. Stephen Hodi, M.D., Wen-Jen Hwu, M.D., Ph.D., Richard Kefford, M.D., Ph.D., \\ Jedd D. Wolchok, M.D., Ph.D., Peter Hersey, M.D., Ph.D., Richard W. Joseph, M.D., \\ Jeffrey S. Weber, M.D., Ph.D., Roxana Dronca, M.D., Tara C. Gangadhar, M.D., \\ Amita Patnaik, M.D., Hassane Zarour, M.D., Anthony M. Joshua, M.B., B.S., Ph.D., \\ Kevin Gergich, M.A., Jeroen Elassaiss-Schaap, Ph.D., Alain Algazi, M.D., \\ Christine Mateus, M.D., Peter Boasberg, M.D., Paul C. Tumeh, M.D., \\ Bartosz Chmielowski, M.D., Ph.D., Scot W. Ebbinghaus, M.D., \\ Xiaoyun Nicole Li, Ph.D., S. Peter Kang, M.D., and Antoni Ribas, M.D., Ph.D.
}

ABSTRACT

From the Angeles Clinic and Research Institute (O.H., P.B.) and the University of California, Los Angeles (P.C.T., B.C., A.R.) - both in Los Angeles; Institute Gustave Roussy, Villejuif, France (C.R., C.M.); University of California, San Francisco, San Francisco (A.D., A.A.); Dana-Farber Cancer Institute, Boston (F.S.H.); University of Texas M.D. Anderson Cancer Center, Houston (W.-J.H.); Westmead Hospital and Melanoma Institute Australia, University of Sydney, Sydney (R.K.), and Kolling Institute, Melanoma Institute of Australia and Newcastle Melanoma Unit, Newcastle, NSW (P.H.) - both in Australia; Memorial Sloan-Kettering Cancer Center, New York (J.D.W.); Mayo Clinic, Jacksonville (R.W.J.), and H. Lee Moffitt Cancer Center, Tampa (J.S.W.) - both in Florida; Mayo Clinic, Rochester, MN (R.D.): Abramson Cancer Center of the University of Pennsylvania, Philadelphia (T.C.G.); South Texas Accelerated Research Therapeutics, San Antonio (A.P.); University of Pittsburgh, Pittsburgh (H.Z.); Princess Margaret Cancer Centre, Toronto (A.M.J.); and Merck Sharp and Dohme, Whitehouse Station, NJ (K.G., J.E.-S., S.W.E., X.N.L., S.P.K.). Address reprint requests to Dr. Ribas at the Department of Medicine, Division of Hematology-Oncology, Jonsson Comprehensive Cancer Center, University of California, Los Angeles, 11934 Factor Bldg., 10833 Le Conte Ave., Los Angeles, CA 90095-1782, or at aribas@ mednet.ucla.edu.

Drs. Hamid, Robert, Daud, Kang, and Ribas contributed equally to this article.

This article was published on June 2, 2013, at NEJM.org.

N EnglJ Med 2013;369:134-44.

DOI: 10.1056/NEJMoal305133

Copyright () 2013 Massachusetts Medical Society.
BACKGROUND

The programmed death 1 (PD-1) receptor is a negative regulator of T-cell effector mechanisms that limits immune responses against cancer. We tested the anti-PD-1 antibody lambrolizumab (previously known as MK-3475) in patients with advanced melanoma.

METHODS

We administered lambrolizumab intravenously at a dose of $10 \mathrm{mg}$ per kilogram of body weight every 2 or 3 weeks or $2 \mathrm{mg}$ per kilogram every 3 weeks in patients with advanced melanoma, both those who had received prior treatment with the immune checkpoint inhibitor ipilimumab and those who had not. Tumor responses were assessed every 12 weeks.

RESULTS

A total of 135 patients with advanced melanoma were treated. Common adverse events attributed to treatment were fatigue, rash, pruritus, and diarrhea; most of the adverse events were low grade. The confirmed response rate across all dose cohorts, evaluated by central radiologic review according to the Response Evaluation Criteria in Solid Tumors (RECIST), version 1.1, was 38\% (95\% confidence interval [CI], 25 to 44), with the highest confirmed response rate observed in the cohort that received $10 \mathrm{mg}$ per kilogram every 2 weeks (52\%; 95\% CI, 38 to 66). The response rate did not differ significantly between patients who had received prior ipilimumab treatment and those who had not (confirmed response rate, 38\% [95\% CI, 23 to 55] and 37\% [95\% CI, 26 to 49], respectively). Responses were durable in the majority of patients (median follow-up, 11 months among patients who had a response); $81 \%$ of the patients who had a response (42 of 52) were still receiving treatment at the time of analysis in March 2013. The overall median progression-free survival among the 135 patients was longer than 7 months.

\section{CONCLUSIONS}

In patients with advanced melanoma, including those who had had disease progression while they had been receiving ipilimumab, treatment with lambrolizumab resulted in a high rate of sustained tumor regression, with mainly grade 1 or 2 toxic effects. (Funded by Merck Sharp and Dohme; ClinicalTrials.gov number, NCT01295827.) 
ANCER EVOLVES TO EXPLOIT MULTIPLE mechanisms in order to avoid immunecell recognition and antitumor effector functions, thereby limiting the clinical benefits of immunotherapy strategies. Antibodies that block the inhibitory receptor cytotoxic T-lymphocyte-associated antigen 4 (CTLA-4), such as ipilimumab, have been shown to release one of these negative immune regulatory pathways, leading to durable responses in a subgroup of patients with metastatic melanoma and an overall survival benefit in patients with metastatic melanoma. ${ }^{1,2}$ The programmed cell death 1 (PD-1) receptor is another inhibitory receptor expressed by $\mathrm{T}$ cells preferentially with long-term exposure to antigens. Its primary ligand, PD-L1 (also known as B7-H1 or CD274), is frequently expressed within the tumor microenvironment, including cancer cells and tumor-infiltrating macrophages. The PD-1 receptor has a second ligand, PD-L2 (also known as B7-DC or CD273), that is preferentially expressed by antigen-presenting cells. ${ }^{3}$ In tumor models, PD- 1 negatively regulates the effector phase of T-cell responses after ligation of PD-L1 expressed within the tumor. ${ }^{4}$ It has been postulated that antibodies that block the interaction between PD-1 and PD-L1 in tumors may preferentially release the cytotoxic function of tumor-specific T cells with fewer systemic toxic effects than those that are seen with other immune checkpoint inhibitors. ${ }^{3,5,6}$

Two large, dose-escalation, phase 1 clinical trials evaluating the safety of the anti-PD- 1 antibody nivolumab (formerly known as BMS936558) and the anti-PD-L1 antibody BMS936559 showed significant antitumor activity in patients with advanced melanoma, lung carcinoma, and renalcell carcinoma, among other cancers, thus validating the PD-1-PD-L1 axis as a therapeutic target. $^{7-9}$ Most tumor responses were durable beyond 1 year. ${ }^{8,9}$ Toxic effects were generally of low grade.

Lambrolizumab (previously known as MK-3475) is a highly selective, humanized monoclonal IgG4-kappa isotype antibody against PD-1 that is designed to block the negative immune regulatory signaling of the PD-1 receptor expressed by $\mathrm{T}$ cells. The variable region sequences of $\mathrm{a}$ very-high-affinity mouse antihuman PD-1 antibody (dissociation constant, 28 pM) were grafted into a human IgG4 immunoglobulin with a stabilizing S228P Fc alteration. The IgG4 immunoglobulin subtype does not engage Fc receptors or activate complement, thus avoiding cytotoxic effects of the antibody when it binds to the T cells that it is intended to activate. In T-cell activation assays that used human donor blood cells, the $50 \%$ effective concentration was in the range of 0.1 to $0.3 \mathrm{nM}$ (unpublished data). The first doseescalation phase 1 study involving patients with solid tumors showed that lambrolizumab was safe at the dose levels tested (1 mg per kilogram of body weight, $3 \mathrm{mg}$ per kilogram, and $10 \mathrm{mg}$ per kilogram, administered every 2 weeks) without reaching a maximum tolerated dose. In addition, clinical responses were observed at all the dose levels. ${ }^{10}$ We report here the safety and antitumor activity of three dosing regimens of lambrolizumab that we evaluated in patients with advanced melanoma.

\section{METHODS}

\section{STUDY OVERSIGHT}

This study was sponsored by Merck Sharp and Dohme, which provided the study drug and worked jointly with the senior academic authors to design the study, collect the data, and interpret the study results. The data were analyzed by a statistician employed by the sponsor and by the senior academic authors. All the authors made the decision to submit the manuscript for publication, vouch for the accuracy and completeness of the data, and attest that the study was conducted as specified in the protocol, which is available with the full text of this article at NEJM.org. The protocol and its amendments were approved by the relevant institutional review boards or ethics committees, and all participants provided written informed consent. All drafts of the manuscript were written by the corresponding author with input from the other authors. The sponsor provided assistance with the preparation of the manuscript. Aside from the authors and those listed in the acknowledgments, no others contributed to the preparation of the manuscript.

\section{STUDY DESIGN}

The primary objective of this study was to evaluate the safety profile of lambrolizumab. The secondary end point was a preliminary analysis of the antitumor activity of lambrolizumab, both in patients who had received prior treatment with ipilimumab and in those who had not. After dose escalation of lambrolizumab to a maximum 
administered dose of $10 \mathrm{mg}$ per kilogram every 2 weeks, ${ }^{10}$ an expansion cohort (Part B of the study) was initiated, with eligibility restricted to patients with advanced melanoma. In Part B of the study, which we report on here, the initial cohort of patients who were enrolled received lambrolizumab as a 30-minute intravenous infusion, every 2 weeks at a dose of $10 \mathrm{mg}$ per kilogram; patients enrolled in additional cohorts in Part B received lambrolizumab as a 30-minute intravenous infusion every 3 weeks at a dose of $2 \mathrm{mg}$ per kilogram or $10 \mathrm{mg}$ per kilogram in sequential or concurrent cohorts without randomization. The study therapy was continued until disease progression was confirmed, unacceptable toxic effects developed, or consent was withdrawn. Patients in whom a scheduled scan showed initial disease progression were allowed to continue receiving treatment until a confirmatory scan was obtained at least 1 month later. Patients underwent a mandatory baseline biopsy and optional biopsies during the course of the trial for biomarker studies. Safety evaluations (clinical and laboratory) were performed at baseline and before each dose of lambrolizumab was administered. No premedications were administered before lambrolizumab infusions. The first scheduled assessment of tumor response was performed 12 weeks after the first dose of lambrolizumab and every 12 weeks thereafter. The evaluation of tumor response was made by investigators at the study site and by a central imaging vendor (Perceptive Informatics).

\section{PATIENTS}

Patients were eligible for participation in Part B of the study if they were 18 years of age or older, had measurable metastatic or locally advanced unresectable melanoma, and had adequate performance status and organ function (according to criteria listed in the protocol). The cohorts of patients who had not received prior treatment with ipilimumab were restricted to patients who had received no more than two prior regimens of systemic therapy. The cohorts of patients who had received prior therapy with ipilimumab included only patients who had full resolution of ipilimumab-related adverse events and no history of severe immune-related adverse events associated with ipilimumab therapy. Patients were allowed to enter the trial 6 weeks after the last dose of ipilimumab was administered. The protocol did not require patients who were asymptomatic to undergo screening brain imaging; however, patients with previously treated brain metastases were required to undergo baseline imaging by means of computed tomographic scanning or magnetic resonance imaging and to have had no evidence of central nervous system progression for 8 weeks. Major exclusion criteria were a melanoma of ocular origin, prior therapy with a PD-1 or PD-L1 blocking agent, current systemic immunosuppressive therapy, or active infections or autoimmune diseases.

\section{PHARMACOKINETIC ANALYSIS}

Peak-level and trough-level blood samples for pharmacokinetic analysis were obtained from patients at the initiation of treatment. Trough samples were also obtained approximately every 12 weeks for the first 12 months of the study and every 6 months thereafter. The serum concentration of lambrolizumab was quantified with the use of a validated electrochemiluminescent assay with a lower limit of quantification of $10 \mathrm{ng}$ per milliliter.

\section{STATISTICAL ANALYSIS}

Data from 135 patients with melanoma who were enrolled and treated according to protocol amendments 02, 03, and 04 were used for the analysis of adverse events. Of the 135 patients, 117 had radiographically measurable disease as assessed by means of central radiologic review and were included in the efficacy analysis of responses according to central review. All other efficacy analyses (an analysis of response on the basis of assessment by the investigator, progression-free survival, and overall survival) were based on data from all 135 patients. Patients were included in the analysis if they received a first dose of study medication by September 6, 2012. Efficacy and safety data that were available as of February 1, 2013, were included in all the analyses. The efficacy analysis included two end points: overall responses derived from investigatorreported data, with assessment according to immune-related response criteria (135 patients) ${ }^{11}$; and overall responses derived from independent, central, blinded radiologic review, with assessment according to the Response Evaluation Criteria in Solid Tumors (RECIST), version 1.1 (117 patients) (see Table S1 in the Supplementary Appendix, available at NEJM.org, for re- 


\begin{tabular}{|c|c|c|c|c|c|c|}
\hline \multirow[t]{3}{*}{ Characteristic } & \multicolumn{2}{|c|}{$10 \mathrm{mg} / \mathrm{kg}$ Every $2 \mathrm{Wk}$} & \multicolumn{2}{|c|}{$10 \mathrm{mg} / \mathrm{kg}$ Every $3 \mathrm{Wk}$} & \multirow{2}{*}{$\begin{array}{c}2 \text { mg/kg Every } 3 \text { Wk } \\
\text { No Prior } \\
\text { Ipilimumab } \\
(\mathrm{N}=22)\end{array}$} & \multirow[t]{2}{*}{ Total $(\mathrm{N}=135)$} \\
\hline & $\begin{array}{l}\text { No Prior } \\
\text { Ipilimumab } \\
(\mathrm{N}=41)\end{array}$ & $\begin{array}{c}\text { Prior } \\
\text { Ipilimumab } \\
(\mathrm{N}=16)\end{array}$ & $\begin{array}{l}\text { No Prior } \\
\text { Ipilimumab } \\
(\mathrm{N}=24)\end{array}$ & $\begin{array}{c}\text { Prior } \\
\text { Ipilimumab } \\
(\mathrm{N}=32)\end{array}$ & & \\
\hline & \multicolumn{6}{|c|}{ number (percent) } \\
\hline \multicolumn{7}{|l|}{ Sex } \\
\hline Male & $23(56)$ & $9(56)$ & $16(67)$ & $17(53)$ & $14(64)$ & $79(59)$ \\
\hline Female & $18(44)$ & $7(44)$ & $8(33)$ & $15(47)$ & $8(36)$ & $56(41)$ \\
\hline \multicolumn{7}{|l|}{ Age (yr) } \\
\hline Mean & 60.4 & 59.4 & 67 & 57.3 & 58.6 & 60.4 \\
\hline Range & $25-94$ & $29-87$ & $37-87$ & $32-77$ & $30-79$ & $25-94$ \\
\hline \multicolumn{7}{|l|}{ Race* } \\
\hline Asian & 0 & 0 & $2(8)$ & 0 & 0 & $2(1)$ \\
\hline White & $41(100)$ & $16(100)$ & $22(92)$ & $32(100)$ & $22(100)$ & $133(99)$ \\
\hline \multicolumn{7}{|l|}{ ECOG performance status $†$} \\
\hline Unknown & $1(2)$ & 0 & 0 & 0 & 0 & $1(1)$ \\
\hline 0 & $32(78)$ & $13(81)$ & $18(75)$ & $21(66)$ & $13(59)$ & $97(72)$ \\
\hline 1 & $8(20)$ & $3(19)$ & $6(25)$ & $11(34)$ & $9(41)$ & $37(27)$ \\
\hline \multicolumn{7}{|l|}{ BRAF mutation status } \\
\hline Mutant & $13(32)$ & $1(6)$ & $1(4)$ & $5(16)$ & $6(27)$ & $26(19)$ \\
\hline Nonmutant & $23(56)$ & $14(88)$ & $21(88)$ & $21(66)$ & $14(64)$ & $93(69)$ \\
\hline Unknown & $5(12)$ & $1(6)$ & $2(8)$ & $6(19)$ & $2(9)$ & $16(12)$ \\
\hline \multicolumn{7}{|l|}{ Brain metastasis } \\
\hline Yes & $3(7)$ & $3(19)$ & 0 & $4(12)$ & $2(9)$ & $12(9)$ \\
\hline No & $38(93)$ & $13(81)$ & $24(100)$ & $28(88)$ & $20(91)$ & $123(91)$ \\
\hline \multicolumn{7}{|l|}{ Lactate dehydrogenase } \\
\hline Normal & $23(56)$ & $11(69)$ & $16(67)$ & $17(53)$ & $13(59)$ & $80(59)$ \\
\hline Elevated $\downarrow$ & $13(32)$ & $5(31)$ & $6(25)$ & $7(22)$ & $5(23)$ & $36(27)$ \\
\hline Unknown & $5(12)$ & 0 & $2(8)$ & $8(25)$ & $4(18)$ & $19(14)$ \\
\hline \multicolumn{7}{|l|}{ M staging of extent of metastasis } \\
\hline$M X$ & 0 & 0 & 0 & $1(3)$ & 0 & $1(1)$ \\
\hline MO & $7(17)$ & $2(12)$ & $2(8)$ & $3(9)$ & $1(5)$ & $15(11)$ \\
\hline $\mathrm{Mla}$ & $1(2)$ & $3(19)$ & $6(25)$ & $3(9)$ & $1(5)$ & $14(10)$ \\
\hline Mlb & $11(27)$ & $3(19)$ & $7(29)$ & $5(16)$ & $2(9)$ & $28(21)$ \\
\hline Mlc & $20(49)$ & $8(50)$ & $9(38)$ & $18(56)$ & $18(82)$ & $73(54)$ \\
\hline Unknown & $2(5)$ & 0 & 0 & $2(6)$ & 0 & $4(3)$ \\
\hline \multicolumn{7}{|l|}{ Previous treatment $\mathbb{\Omega}$} \\
\hline No prior systemic treatment & $16(39)$ & 0 & $12(50)$ & 0 & $14(64)$ & $42(31)$ \\
\hline $\begin{array}{l}\text { Immunotherapy, excluding } \\
\text { ipilimumab }\end{array}$ & $11(27)$ & $4(25)$ & $5(21)$ & $10(31)$ & $4(18)$ & $34(25)$ \\
\hline Chemotherapy & $11(27)$ & $8(50)$ & $9(38)$ & $14(44)$ & $5(23)$ & $47(35)$ \\
\hline BRAF inhibitor & $4(10)$ & 0 & $1(4)$ & $4(12)$ & $1(5)$ & $10(7)$ \\
\hline
\end{tabular}

* Race was self-reported.

$\uparrow$ An Eastern Cooperative Oncology Group (ECOG) performance status of 0 indicates that the patient is fully active, 1 that the patient is restricted in physically strenuous activity but ambulatory and able to carry out work of a light or sedentary nature, and 2 that the patient is ambulatory and capable of all self-care but unable to carry out any work activities.

$\mp$ An elevated level was considered to be a level higher than the upper limit of the normal range.

$\int$ This category included treatments for advanced disease. The numbers may add up to more than $100 \%$ since a patient may have received more than one type of oncologic therapy.

The New England Journal of Medicine 
Table 2. Drug-Related Adverse Events.*

\begin{tabular}{|c|c|c|}
\hline \multirow[t]{2}{*}{ Drug-Related Event } & $\begin{array}{c}\text { All Grades } \\
(\mathrm{N}=135)\end{array}$ & $\begin{array}{c}\text { Grade } 3 \text { or } 4 \\
(N=135)\end{array}$ \\
\hline & \multicolumn{2}{|c|}{ number (percent) } \\
\hline Any & 107 (79) & $17(13)$ \\
\hline Hypothyroidism & $11(8)$ & $1(1)$ \\
\hline \multicolumn{3}{|l|}{ Gastrointestinal disorder } \\
\hline Diarrhea & $27(20)$ & $1(1)$ \\
\hline Nausea & $13(10)$ & 0 \\
\hline Abdominal pain & $7(5)$ & $1(1)$ \\
\hline \multicolumn{3}{|l|}{ Generalized symptom } \\
\hline Fatigue & $41(30)$ & $2(1)$ \\
\hline Myalgia & $16(12)$ & 0 \\
\hline Headache & $14(10)$ & 0 \\
\hline Asthenia & $13(10)$ & 0 \\
\hline Pyrexia & $10(7)$ & 0 \\
\hline Chills & $9(7)$ & 0 \\
\hline Decreased appetite & $6(4)$ & $1(1)$ \\
\hline \multicolumn{3}{|c|}{ Increase in aminotransferase level } \\
\hline AST & $13(10)$ & $2(1)$ \\
\hline ALT & $11(8)$ & 0 \\
\hline Renal failure & $3(2)$ & $2(1)$ \\
\hline \multicolumn{3}{|l|}{ Respiratory disorder } \\
\hline Cough & $11(8)$ & 0 \\
\hline Dyspnea & $6(4)$ & 0 \\
\hline Pneumonitis & $6(4)$ & 0 \\
\hline \multicolumn{3}{|l|}{ Skin disorder } \\
\hline Rash & $28(21)$ & $3(2)$ \\
\hline Pruritus & $28(21)$ & $1(1)$ \\
\hline Vitiligo & $12(9)$ & 0 \\
\hline
\end{tabular}

* Included are drug-related adverse events that occurred in at least five patients or drug-related grade 3 or 4 adverse events that occurred in at least two patients. ALT denotes alanine aminotransferase, and AST aspartate aminotransferase.

sponse criteria). ${ }^{12}$ The overall response rate was defined as the number of patients with a complete or partial response divided by the total number of patients who had measurable disease at baseline and received at least one treatment dose. The overall response rate and exact twosided $95 \%$ confidence interval were calculated. Toxic effects were graded with the use of the National Cancer Institute Common Terminology Criteria for Adverse Events, version 4.0. ${ }^{13}$ De- scriptive statistics were provided for the pharmacokinetic analysis of trough and peak samples according to treatment cohort.

\section{RESULTS}

\section{BASELINE CHARACTERISTICS OF THE PATIENTS}

Between December 1, 2011, and September 6, 2012, a total of 135 patients with advanced melanoma were enrolled in this multi-institutional, international, phase 1 expansion study. Initially, patients were enrolled in a cohort that received lambrolizumab at a dose of $10 \mathrm{mg}$ per kilogram every 2 weeks. Subsequently, additional patients were enrolled in concurrent (not randomized) cohorts that received lambrolizumab at $10 \mathrm{mg}$ per kilogram or $2 \mathrm{mg}$ per kilogram every 3 weeks. A distinction was made between patients who had received prior treatment with ipilimumab (48 patients) and those who had not (87 patients) to provide preliminary data on the safety and antitumor activity of lambrolizumab on the basis of prior or no prior treatment with ipilimumab. The median time between the last dose of ipilimumab and the initiation of lambrolizumab was 23 weeks (range, 6 to 83). The majority of patients (38 of 48) were enrolled more than 12 weeks after the last dose of ipilimumab, and $90 \%$ (43 of 48) had received three or more infusions of ipilimumab. The baseline characteristics of the patients were similar across all the treatment groups (Table 1). Overall, more than $50 \%$ of the patients had visceral metastases (stage M1c), approximately $25 \%$ had an elevated lactate dehydrogenase level, and close to $9 \%$ had a history of brain metastases - all of which are recognized as poor prognostic factors in patients with advanced melanoma.

\section{SAFETY}

Table 2 shows the adverse events that were considered to be related to lambrolizumab therapy. Table S2 in the Supplementary Appendix provides further details of drug-related toxic effects according to the dosing cohort, and Table S3 in the Supplementary Appendix describes all adverse events regardless of the cause, according to the dosing cohort. Of the 135 patients who received at least one dose of lambrolizumab, 79\% reported drug-related adverse events of any grade, and 13\% reported grade 3 or 4 drug-related ad- 
verse events. Generalized symptoms, including fatigue and asthenia, fever and chills, myalgias, and headaches, were reported frequently but were of low grade in more than $95 \%$ of the cases. In addition to the data shown in the tables, there was one case of grade 1 infusion reaction. Rashes and pruritus were reported in $21 \%$ of the patients; grade 3 or 4 pruritus was reported in 1\% of the patients, and grade 3 or 4 rash in $2 \%$. Vitiligo was attributed to lambrolizumab in $9 \%$ of the patients. The highest incidence of overall treatment-related adverse events was seen among the patients who received $10 \mathrm{mg}$ of lambrolizumab per kilogram every 2 weeks, as compared with the patients receiving $10 \mathrm{mg}$ per kilogram every 3 weeks and those receiving 2 mg per kilogram every 3 weeks (23\%, vs. $4 \%$ and $9 \%$, respectively) (Table S2 in the Supplementary Appendix).

Adverse events of particular interest were of an inflammatory or autoimmune nature. Treatment-related pneumonitis was reported in $4 \%$ of the patients; none of the cases were grade 3 or 4. One patient, a 96-year-old man, died during the course of the study. Initial asymptomatic pneumonitis was identified on a scan, and lambrolizumab was discontinued. Subsequently, after shortness of breath developed, the patient received glucocorticoids. The clinical course was complicated when acute bronchopneumonia and pneumothorax due to bronchoscopy and biopsies were diagnosed. Although the pulmonary infiltrates were reduced with glucocorticoids, the patient died from a myocardial infarction and bronchopneumonia. Grade 3 or 4 elevations of aminotransferase levels were reported in $1 \%$ of the patients. Two cases of grade 3 renal failure were reported. Both cases were potentially immune-mediated, and the patients' renal function improved with glucocorticoid therapy along with the discontinuation of lambrolizumab. Although diarrhea was reported in $20 \%$ of the patients, a single case of grade 3 treatment-related diarrhea was reported. This case was managed with treatment of the symptoms, and the patient recovered promptly without glucocorticoid treatment. Hypothyroidism was reported in $8 \%$ of the patients and was effectively managed with thyroid-replacement therapy. In addition to the data shown in the tables, grade 3 hyperthyroidism and grade 2 adrenal insufficiency developed
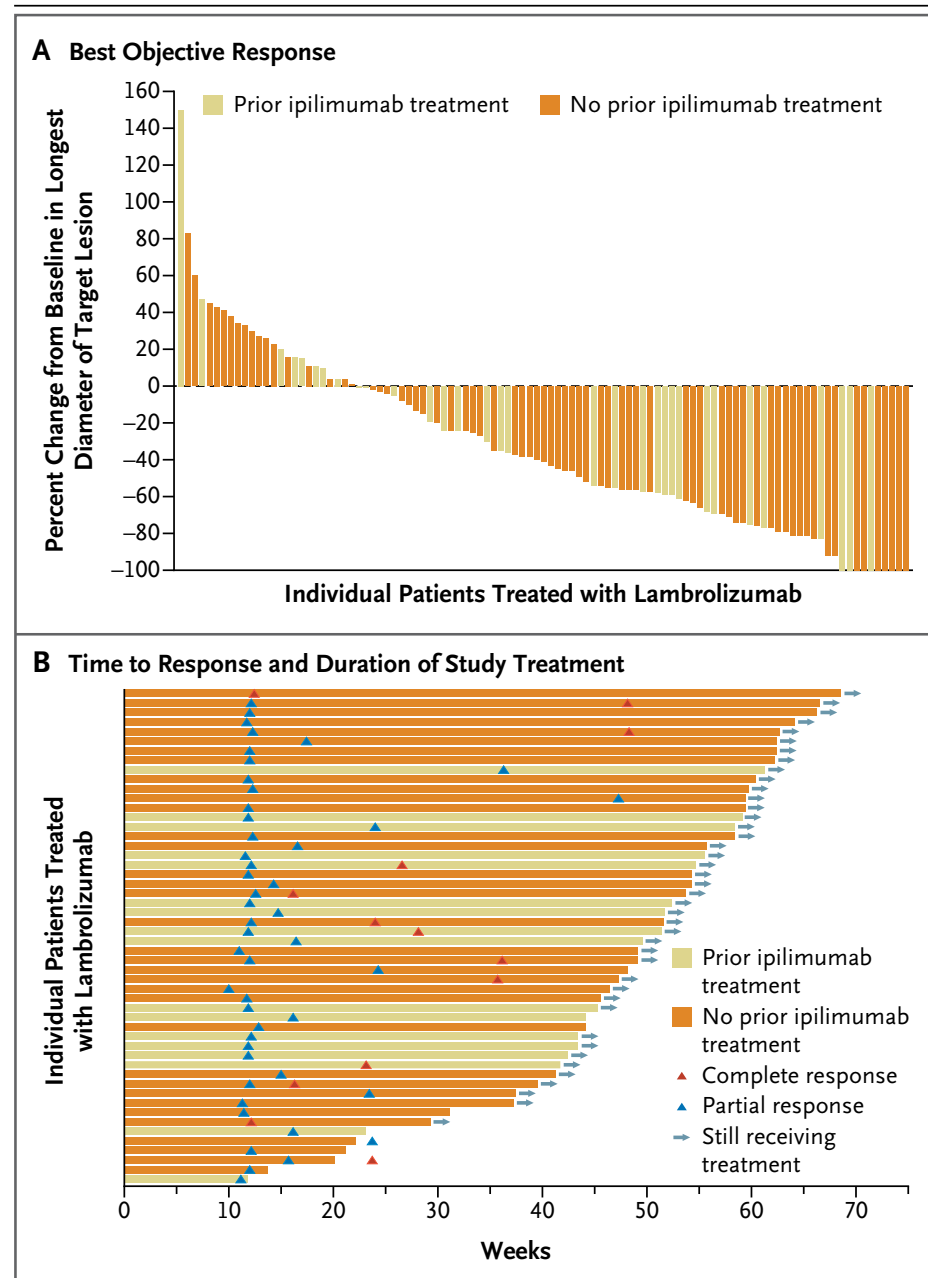

Figure 1. Antitumor Activity of Lambrolizumab.

Data on the antitumor activity of lambrolizumab, as assessed by independent, central radiologic review, is shown for the patients who could be evaluated. Panel A shows a waterfall plot of the best objective response according to prior treatment with ipilimumab, measured as the maximum change from baseline in the sum of the longest diameter of each target lesion. A total of 10 of 103 patients with radiographically measurable disease at baseline and at least one evaluation after treatment had a $100 \%$ reduction in target lesions. Panel B shows the time to response and the duration of study treatment. A total of 42 of the 52 patients who had a response were still receiving the study treatment at the time of the current analysis. Of the 10 patients who discontinued therapy, 5 discontinued owing to toxic effects, and 2 of these patients showed improvement in their response after discontinuation (denoted by the two triangles that are outside the bar of the on-treatment period).

in one patient; these were managed with standard measures, and the patient continued in the study with a durable response. No other endocrinopathies were recorded. 


\begin{tabular}{|c|c|c|c|c|c|c|}
\hline \multirow[t]{2}{*}{ Regimen and Ipilimumab Status } & \multicolumn{4}{|c|}{ RECIST } & \multicolumn{2}{|c|}{ Immune-Related Response } \\
\hline & $\begin{array}{c}\text { No. of } \\
\text { Patients }\end{array}$ & $\begin{array}{c}\text { Confirmed and } \\
\text { Unconfirmed } \\
\text { Objective Response } \\
\text { no. (\% [ }\end{array}$ & $\begin{array}{l}\text { Confirmed } \\
\text { Objective Response } \\
95 \% \mathrm{Cl}] \text { ) }\end{array}$ & $\begin{array}{c}\text { Duration of } \\
\text { Response' } \\
\text { mo }\end{array}$ & $\begin{array}{c}\text { No. of } \\
\text { Patients }\end{array}$ & $\begin{array}{c}\text { Confirmed } \\
\text { Objective Response } \\
\text { no. (\% }[95 \% \mathrm{Cl}] \text { ) }\end{array}$ \\
\hline \multicolumn{7}{|l|}{$10 \mathrm{mg} / \mathrm{kg}$ every $2 \mathrm{wk}$} \\
\hline No prior ipilimumab & 39 & 21 (54 [37-70]) & $19(49[32-65]) \div$ & $1.9-10.8$ & 41 & $23(56[40-72])$ \\
\hline Prior ipilimumab & 13 & $8(62[32-86])$ & $8(62[32-86]) \mathbb{\int}$ & $2.8-8.3$ & 16 & $9(56[30-80])$ \\
\hline Total & 52 & $29(56[41-69])$ & $27(52[38-66])$ & $1.9-10.8$ & 57 & $32(56[42-69])$ \\
\hline \multicolumn{7}{|l|}{$10 \mathrm{mg} / \mathrm{kg}$ every $3 \mathrm{wk}$} \\
\hline No prior ipilimumab & 19 & $7(37[16-62])$ & $5(26[9-51])$ & $2.6-5.6$ & 24 & $8(33[16-55])$ \\
\hline Prior ipilimumab & 26 & $9(35[17-56])$ & $7(27[12-48])$ & $2.8-8.3$ & 32 & 7 (22 [9-40]) \\
\hline Total & 45 & $16(36[22-51])$ & $12(27[15-42])$ & $2.6-8.3$ & 56 & 15 (27 [16-40]) \\
\hline $\begin{array}{l}2 \mathrm{mg} / \mathrm{kg} \text { every } 3 \mathrm{wk} \text {, no prior } \\
\text { ipilimumab }\end{array}$ & 20 & 7 (35 [15-59]) & $5(25[9-49]) 9$ & $2.1-5.5$ & 22 & $3(14[3-35])$ \\
\hline Total\| & 117 & $52(44[35-54]) * *$ & $44(38[25-44])$ & $1.9-10.8$ & 135 & $50(37[29-45])$ \\
\hline
\end{tabular}

* The efficacy population of patients with measurable disease was assessed by means of an independent, central, blinded radiologic review with the use of the Response Evaluation Criteria in Solid Tumors (RECIST), version 1.1, and by means of investigator assessment with the use of immune-related response criteria. The latter was the primary end point of the study. Responses based on immune-related response criteria included only those that were confirmed on two consecutive scans obtained at least 28 days apart.

$\dagger$ The duration of response was defined as the time from the first response to the time of documented progression or, in the case of censored data, the most recent tumor assessment. All the lower and upper ranges listed here are for censored data and refer to the time from the first response to the most recent tumor assessment, except for the lower range in the group with no prior ipilimumab, as well as the total cohort, receiving $10 \mathrm{mg}$ per kilogram of body weight every 3 weeks; these two lower ranges refer to the time from first response to the time of documented progression. Only confirmed responses were included in the calculation of duration of response.

$¥$ Three of these patients had a complete response.

$\int$ Two of these patients had a complete response.

I One of these patients had a complete response.

$\|$ The confirmed response rate, according to RECIST, version 1.1, was $38 \%(95 \% \mathrm{Cl}, 23$ to 55$)$ among patients who had received prior ipilimumab treatment and $37 \%(95 \% \mathrm{Cl}, 26$ to 49$)$ among patients who had not received prior ipilimumab treatment.

** Six patients with initial responses were awaiting confirmation of the response at the time of the data cutoff for this report. One response has since been confirmed, but since it was confirmed after the data cutoff for the current analysis, the data on overall response rate have not been modified.

\section{PHARMACOKINETICS}

Serum concentrations of lambrolizumab in samples obtained before and after administration of the drug were lower by a factor of approximately 5 in patients receiving $2 \mathrm{mg}$ per kilogram every 3 weeks than in those receiving $10 \mathrm{mg}$ per kilogram every 3 weeks; steady-state trough concentrations were $20 \%$ greater in the patients receiving $10 \mathrm{mg}$ per kilogram every 2 weeks than in those receiving the same dose every 3 weeks (Table S4 in the Supplementary Appendix). The increase in trough serum concentrations over time is consistent with the half-life of lambrolizumab of about 2 to 3 weeks. ${ }^{10}$

\section{CLINICAL ACTIVITY}

We evaluated the response to therapy using two different criteria: investigator-assessed immunerelated response criteria, which were designed to analyze the response to immunotherapy agents ${ }^{11}$; and RECIST, ${ }^{12}$ as assessed by independent, central radiologic review, which is used routinely to assess responses to cytotoxic agents for cancer. The overall response rate during receipt of therapy, across all doses, on the basis of assessment by the investigator according to immune-related response criteria was $37 \%$. The confirmed response rate across all doses, as assessed by central review according to RECIST, was 38\% (44 of 
117 patients). There were an additional 8 unconfirmed responses. Six of these unconfirmed responses were in patients who had not yet undergone confirmatory scanning at the time of the data cutoff. Since then, 1 of these patients has been confirmed as having an objective response. The response rate, including confirmed and unconfirmed responses, across all doses was $44 \%$ (44 confirmed and 8 unconfirmed). The confirmed response rate, as assessed by central review according to RECIST, ranged from $25 \%$ in the cohort that received $2 \mathrm{mg}$ per kilogram every 3 weeks to $52 \%$ in the cohort that received $10 \mathrm{mg}$ per kilogram every 2 weeks. As shown in Figure $1 \mathrm{~A}, 77 \%$ of the patients had a reduction in the tumor burden during the study, including 8 patients who were confirmed by central review as having stable disease for longer than 24 weeks. Responses did not vary according to prior exposure to ipilimumab (Table 3 and Fig. 1A).

Figure $1 \mathrm{~B}$ shows the time to response and the treatment duration in the 52 patients who had an objective response (confirmed or unconfirmed) on the basis of central radiologic review according to RECIST. The majority of responses were seen at the time the first imaging was performed at 12 weeks. An additional 17 patients who had stable disease at an early assessment showed durable objective response with continued treatment, with 1 patient achieving a partial response according to RECIST after 48 weeks of treatment. The median duration of response had not been reached at the time of the analysis, at a median follow-up time of 11 months. A total of $81 \%$ of the patients who had a response were still receiving the study treatment at the time of the analysis in March 2013. Of the 52 patients with a response, 5 discontinued treatment owing to disease progression, and 5 discontinued treatment for other reasons (most commonly adverse events). The median progression-free survival among the 135 patients, as estimated with the use of a Kaplan-Meier analysis, was more than 7 months. The estimated median overall survival had not been reached.

Biopsied specimens of regressing lesions were densely infiltrated by CD8+ cytotoxic T lymphocytes (Fig. 2A and 2B), a finding that is consistent with the mechanism of action of lambrolizumab. As shown in Figures 2C and 2D, some patients may have had delayed responses after an initial period in which the tumor burden increased, a process consistent with an immunerelated response.

\section{DISCUSSION}

Immunotherapeutic agents, including high-dose interleukin-2, interferon alfa, and anti-CTLA-4 antibodies, have shown activity in patients with advanced melanoma; however, this is an infrequent event that is seen in 10 to $15 \%$ of patients. ${ }^{6,14}$ This study provides evidence of a high response rate with lambrolizumab in patients with advanced melanoma. Most responses to lambrolizumab were durable - similar to the pattern of response with other immunotherapies ${ }^{1,2,15,16}$ and the majority of responses were ongoing at the time of the current analysis. The cohort with the maximum administered dose of lambrolizumab (10 mg per kilogram every 2 weeks) showed the highest response rate of $52 \%$. This cohort also showed the highest rate of drug-related adverse events, although that may be due in part to a longer duration of therapy (Table S5 in the Supplementary Appendix). It is also possible that these nonrandomized cohorts had unmeasured confounders that could have led to different outcomes - although this is not readily apparent from an analysis of the baseline characteristics of the patients. Therefore, an additional randomized expansion of the cohort is ongoing to investigate the higher response rate observed in the cohort receiving $10 \mathrm{mg}$ per kilogram every 2 weeks as compared with every 3 weeks.

Although cross-study comparisons of adverseevent rates should be viewed with caution, it seems that in comparison with anti-CTLA-4 therapy, lambrolizumab therapy was associated with a lower incidence and a different spectrum of immune-related adverse events, possibly owing to a distinct mechanism of action with a more targeted effect on tumor-specific T cells. ${ }^{6}$

Prior exposure to other immunotherapy strategies, most notably the use of the anti-CTLA-4 antibody ipilimumab or interleukin-2, did not have a major effect on the benefit from lambrolizumab treatment. Furthermore, the rate of immune-mediated or other toxic effects was not increased in patients who had received prior 


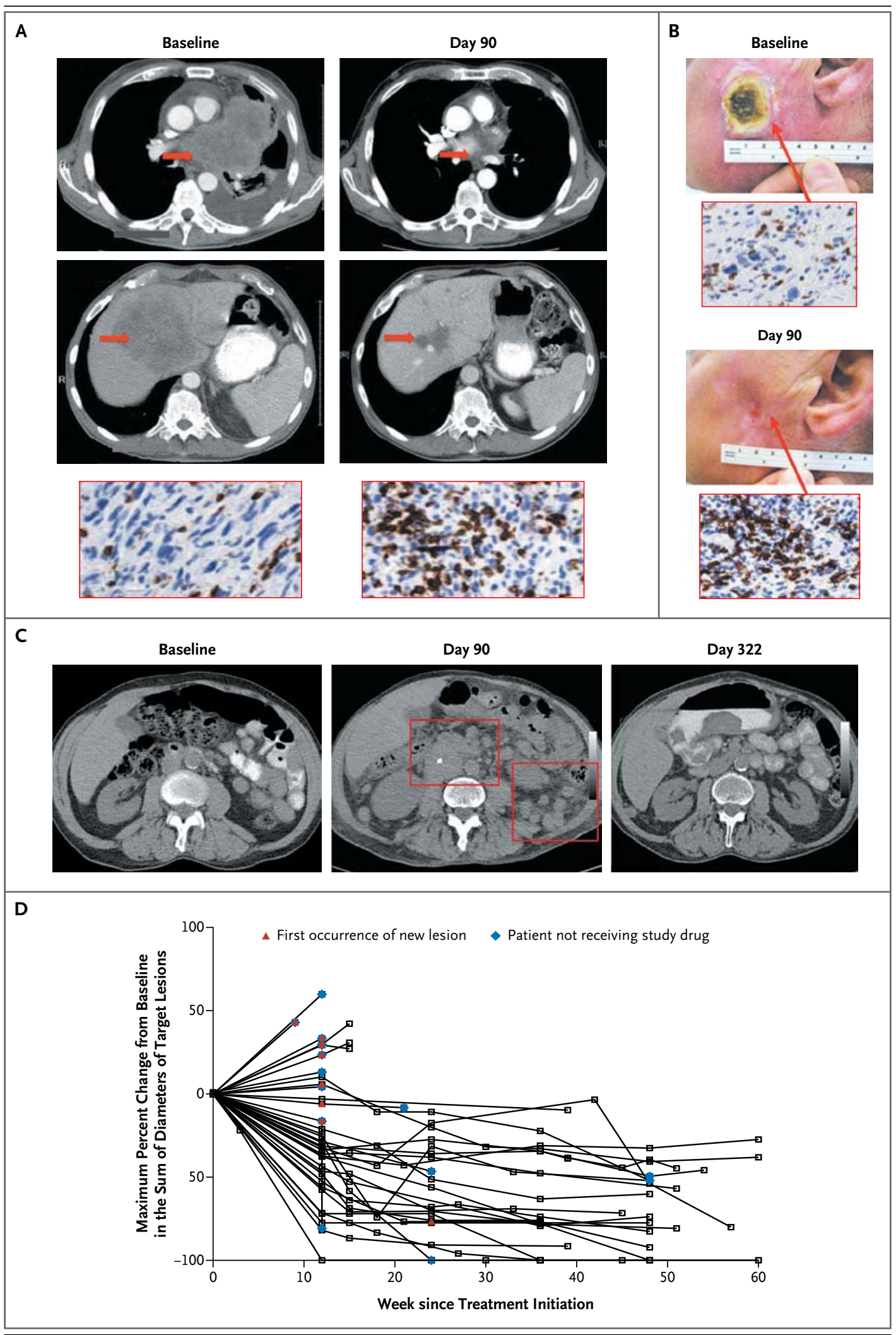

The New England Journal of Medicine 
Figure 2 (facing page). Tumor Responses with Lambrolizumab.

Shown are examples of tumor responses in patients treated with lambrolizumab. Panel A shows images obtained from a patient with BRAF nonmutant metastatic melanoma who had symptomatic progression after biochemotherapy and treatment with high-dose interleukin-2 and ipilimumab; the patient had rapid resolution of symptoms and showed a partial response with lambrolizumab at the initial imaging on day 90 . Arrows point to sites of melanoma metastases in the lung and liver. Immunohistochemical staining of biopsied specimens obtained before and after treatment show an increased CD8 T-cell infiltrate after treatment. Panel B shows the resolution of a local relapse of desmoplastic melanoma in a patient who had not received prior treatment with ipilimumab; an additional tumor response was observed in nodal and lung metastases (not shown). CD8 immunohistochemical staining of biopsy specimens obtained before and after treatment shows increased CD8 T-cell infiltrate. Panel C shows images from a patient without prior treatment with ipilimumab who had metastatic mucosal melanoma with significant progression at the initial 12-week imaging (red boxes), at which time lambrolizumab was discontinued. Without receiving any other therapy, the patient went on to have a nearly complete response that is ongoing more than 1 year after the start of the study. Panel D is a plot of the change in tumor burden (assessed as the longest dimension of the lesion) over time in patients with melanoma who had not received prior treatment with ipilimumab and who received lambrolizumab at a dose of $10 \mathrm{mg}$ per kilogram of body weight every 2 weeks. In most patients who had an objective response, the responses were durable and were evident at the initial evaluation ( 12 weeks). Tumor regression followed both conventional and immunerelated patterns of response, such as a prolonged reduction in the tumor burden in the presence of new lesions.

treatment with ipilimumab. In addition, a response to lambrolizumab was documented in patients who had previously had disease progression while receiving other forms of immuno- therapy, chemotherapy, or BRAF-targeted therapy. The striking anticancer activity observed with lambrolizumab requires confirmation in larger studies. A randomized clinical trial involving patients who have ipilimumab-refractory disease (and if positive for the BRAF V600 mutation have received treatment with an approved BRAF or MEK inhibitor) has commenced.

The ability to induce immune responses against cancer by abrogating an immune-system checkpoint that limits the antitumor activity of preexisting tumor-specific cytotoxic $\mathrm{T}$ cells points to the importance of focusing on immune regulatory events for cancer therapy. As first described with anti-CTLA-4 antibodies in preclinical studies ${ }^{17}$ and in patients, ${ }^{1,2,16}$ this study confirms the importance of releasing inhibitory immune regulation by PD- 1 for effective antitumor immunity. ${ }^{6}$

Supported by Merck Sharp and Dohme.

Disclosure forms provided by the authors are available with the full text of this article at NEJM.org.

We thank Dr. Mary E. Hanson, Martha C. Vollmer, and Margaret Hodgson (Merck Sharp and Dohme) for assistance with preparation of the manuscript; Dr. Eric Rubin, Dr. Alise Reicin, Dr. Robert Iannone, Dr. Joseph E. Eid, Dr. Cong Chen, Anne Morosky, Maxine Giannotti, Kellie Celentano, and Amanda McDonald (Merck Sharp and Dohme) for critical review of the manuscript; Dr. Andrea Perrone and Linda Gammage (Merck Sharp and Dohme) for data collection and interpretation; and the following investigators and site personnel: Dr. John Glaspy, Denise Oseguera, Elizabeth Seja, Christine Kivork, Derek Chung, and Antonio J. Gutierrez (University of California, Los Angeles); Dr. Emilie Routier and Severine Roy (Institute Gustave Roussy); Dr. Georgina Long and Arthur Clements (Westmead Hospital and Melanoma Institute Australia, University of Sydney); Dr. Naiyer Rizvi and Nathan Gray (Memorial Sloan-Kettering Cancer Center); Ann Cross (Mayo Clinic, Jacksonville); Dr. Ragini Kudchadkar (H. Lee Moffitt Cancer Center); Dr. Alan H. Bryce and Dr. Peter Cohen (Mayo Clinic, Scottsdale); Joseph Ilagan, Karen Perdon, and Portia Velasquez (M.D. Anderson Cancer Center); David Hogg and Marcus Butler (Princess Margaret Cancer Centre); Dr. Anthony Tolcher, Dr. Drew Rasco, Dr. Kyriakos Papadopoulos, Dr. Muralidhar Beeram, Guillermo Espino, and Crystal Perez (South Texas Accelerated Research Therapeutics); Dr. John Kirkwood and Dr. Hussein Tawbi (University of Pittsburgh); and Dr. Lynn Schuchter, Dr. Ravi Amaravadi, Mary Carberry, Lydia Giles, and Naomi Haas (University of Pennsylvania).

\section{REFERENCES}

1. Hodi FS, O'Day SJ, McDermott DF, et al. Improved survival with ipilimumab in patients with metastatic melanoma. N Engl J Med 2010;363:711-23.

2. Robert C, Thomas L, Bondarenko I, et al. Ipilimumab plus dacarbazine for previously untreated metastatic melanoma. N Engl J Med 2011;364:2517-26.

3. Pardoll DM. The blockade of immune checkpoints in cancer immunotherapy. Nat Rev Cancer 2012;12:252-64.
4. Blank C, Brown I, Peterson AC, et al. PD-L1/B7H-1 inhibits the effector phase of tumor rejection by $\mathrm{T}$ cell receptor (TCR) transgenic CD8+ T cells. Cancer Res 2004;64:1140-5.

5. Okazaki T, Honjo T. PD-1 and PD-1 ligands: from discovery to clinical application. Int Immunol 2007;19:813-24.

6. Ribas A. Tumor immunotherapy directed at PD-1. N Engl J Med 2012;366: 2517-9.
7. Brahmer JR, Drake CG, Wollner I, et al. Phase I study of single-agent anti-programmed death-1 (MDX-1106) in refractory solid tumors: safety, clinical activity, pharmacodynamics, and immunologic correlates. J Clin Oncol 2010;28:3167-75.

8. Brahmer JR, Tykodi SS, Chow LQ, et al. Safety and activity of anti-PD-L1 antibody in patients with advanced cancer. N Engl J Med 2012;366:2455-65.

9. Topalian SL, Hodi FS, Brahmer JR, et al. 
Safety, activity, and immune correlates of anti-PD-1 antibody in cancer. N Engl J Med 2012;366:2443-54.

10. Patnaik A, Kang SP, Tolcher AW, et al. Phase I study of MK-3475 (anti-PD-1 monoclonal antibody) in patients with advanced solid tumors. J Clin Oncol 2012; 30:Suppl:2512. abstract.

11. Wolchok JD, Hoos A, O'Day S, et al. Guidelines for the evaluation of immune therapy activity in solid tumors: immunerelated response criteria. Clin Cancer Res 2009;15:7412-20.

12. Eisenhauer EA, Therasse P, Bogaerts J, et al. New response evaluation criteria in solid tumours: revised RECIST guideline (version 1.1). Eur J Cancer 2009;45:228-47. 13. CTCAE v4. 2013 (http://ctep.cancer .gov/protocolDevelopment/electronic_ applications/ctc.htm).

14. Ribas A, Butterfield LH, Glaspy JA, Economou JS. Current developments in cancer vaccines and cellular immunotherapy. J Clin Oncol 2003;21:2415-32.

15. Atkins MB, Lotze MT, Dutcher JP, et al. High-dose recombinant interleukin 2 therapy for patients with metastatic melanoma: analysis of 270 patients treated between 1985 and 1993. J Clin Oncol 1999;17:2105-16.

16. Ribas A, Kefford R, Marshall MA, et al. Phase III randomized clinical trial comparing tremelimumab with standard-ofcare chemotherapy in patients with advanced melanoma. J Clin Oncol 2013;31: 616-22.

17. Leach DR, Krummel MF, Allison JP. Enhancement of antitumor immunity by CTLA-4 blockade. Science 1996;271: 1734-6.

Copyright (c) 2013 Massachusetts Medical Society 